\title{
Efecto de la harina de champiñón y flavofosfolipol sobre la canal en pollos de engorda
}

\section{Effect of mushroom powder and flavophospholipol on carcass in broiler chickens}

\author{
Sepideh Shamsia, Alireza Seidavia, Maliheh Rahatia, J osé Ángel Gómez Nietob
}

\begin{abstract}
RESUMEN
Tras demostrar efectos beneficiosos sobre el desarrollo del crecimiento de pollos, se realizó un estudio para evaluar el efecto de la adición de flavofosfolipol y harina de champiñón común desde el nacimiento y durante todo el período de crecimiento sobre las características de la canal, peso de vísceras y medidas del tracto digestivo en pollos de engorda. Se utilizaron 300 pollitos machos de un día de edad distribuidos al azar en 10 tratamientos con tres repeticiones por tratamiento y 10 pollitos por corral. El experimento consistió en un arreglo factorial de 2 x 5: cinco concentraciones de harina de champiñón (0, 0.5, 1.0, 1.5 y $2.0 \mathrm{~g} / \mathrm{kg}$ de dieta) y la adición de dos concentraciones de flavofosfolipol $(0.0$ o $5.0 \mathrm{mg} / \mathrm{kg})$, desde el nacimiento hasta el sacrificio a los 42 días de edad. Los resultados demostraron que la adición de harina de champiñón y flavofosfolipol tienen un efecto positivo sobre el peso de partes de la canal de importancia comercial como el pecho y muslos. En cambio, no hubo ningún efecto sobre el peso de las alas. En relación al peso de las vísceras, la harina de champiñón (pero no el flavofosfolipol o la interacción de ambos), afectó el peso del timo, bazo, bolsa de Fabricio e hígado. Finalmente, a pesar de escasos resultados significativos en varias medidas del tracto digestivo, no se encontró ningún efecto estadístico de la harina de champiñón o la adición del antibiótico en estas variables.
\end{abstract}

PALABRAS CLAVE: Pollos, Canal, Flavofosfolipol, Agaricus bisporus, Tracto digestivo.

\begin{abstract}
After showed its beneficial effects on chicken growth performance, a study was conducted to evaluate the effect of adding from birth and during the whole growth period, flavophospholipol and edible mushrooms powder on carcass characteristics, viscera weights and digestive tract measurements of male broiler chicken. A total of 300 one-dayold male broiler chicks were randomly distributed into 1 to 10 treatments with three replicates per treatment and 10 chicks per pen. The experiment consisted in a $2 \times 5$ factorial arrangement of treatments including five concentrations of mushrooms powder $(0,0.5,1.0,1.5$ and $2.0 \mathrm{~g} / \mathrm{kg}$ of diet) and the addition of $0.0 \mathrm{or} 5.0 \mathrm{mg} / \mathrm{kg}$ of flavophospholipol from birth date to slaughter time at $42-d$. Results show that the additions of dietary mushroom powder and flavophospholipol have a positive effect on weights of carcass parts with commercial importance as the breast and drumsticks. In contrast, there is no effect on wings weight. Regarding viscera weights, mushroom powder but not flavophospholipol or the interaction of both, affected the thymus, spleen, bursa of Fabricius and liver weights. Finally, despite weak statistically significant results on several measurements of the digestive tract, results show no effect of mushroom powder or the antibiotic addition on these variables.
\end{abstract}

KEY WORDS: Broiler, Carcass, Flavophospholipol, Agaricus bisporus, Digestive tract.

El sector ganadero en Asia se encuentra bajo presión para adaptarse y ampliarse(1). Estas adaptaciones se refieren esencialmente a un
The livestock sector in Asia is under pressure to adapt and expand(1). These adaptations involve mainly a shift in livestock species and

Recibido el 9 de diciembre de 2014. Aceptado el 19 de febrero de 2015

a Department of Animal Science, Rasht Branch, Islamic Azad University, Rasht, Iran. Telephone: +989113313073. alirezaseidavi@iaurasht.ac.ir. Correspondencia al segundo autor.

b Consejería Agricultura Castilla-La Mancha. Oficina Comarcal Agraria, 02630, La Roda (Albacete), España. 
cambio en el tipo de especies y características de la ganadería, con el mayor cambio dirigido al aumento en el número de especies de monogástricos (cerdos y aves de corral). La industria avícola nunca ha estado bajo una presión mayor que en el momento presente(2). La producción de aves de corral en Irán ha ido en aumento cada año a una tasa del $8 \%$ en la última década, alcanzando 1'589,000 cabezas y producción de 1.9 t en $2011^{(3)}$, que es la más grande de Oriente Medio.

El champiñón común (Agaricus bisporus) con un alto contenido en polifenoles, ergotioneina, vitaminas, minerales y polisacáridos(4) ha demostrado que posee propiedades biológicas valiosas, incluyendo actividad antitumoral, antiaromatasa, antimicrobiana, inmunomoduladora, anti-inflamatoria y antioxidante(5-11). A pesar de los efectos positivos conocidos sobre la salud humana(12,13), hay poca información sobre el efecto del consumo de hongos en pollos. Sin embargo, en pavos, se ha demostrado que A. bisporus, debido al contenido en polisacáridos, tiene un efecto similar al de un probiótico y que mejora la eficiencia en la digestibilidad y la utilización de forrajes $(14,15)$.

La producción Iraní de setas y trufas ha aumentado de 10,000 t en 1997 a 82,000 t en 2011 (estimación de la FAO), manteniendo en este año el octavo lugar en la producción mundial(3). La industria de producción de hongos genera cantidades significativas de desperdicios que se eliminan durante el proceso de producción. El reciclaje de este subproducto como aditivo alimentario para la producción de pollos de engorda podría aumentar la eficiencia de la industria alimentaria de Irán mediante la reducción de costos de producción.

El impacto positivo del uso de antibióticos como promotores del crecimiento (AGP, por sus siglas en inglés) debido a la supresión de microorganismos dañinos(16) y a la inhibición de efectos inflamatorios es bien conocido en la industria de aves de corral (17). La asociación del aumento de la resistencia bacteriana a los functions, with the greatest change being an increase in the number of monogastric animals (pig and poultry). The poultry industry has never been under a greater pressure than at the present time(2). The production of poultry in Iran has been increasing annually at a rate of $8 \%$ for the last decade reaching 1.589 million heads and 1.9 t of output(3) in 2011, which is the largest in the Middle East.

The white button mushroom (Agaricus bisporus) with high content of polyphenols, ergothioneine, vitamins, minerals and polysaccharides(4) has been demonstrated to possess valuable biological properties including antitumor, antiaromatase, antimicrobial, immunomodulatory, anti-inflammatory, and antioxidant activities(511). Despite the largely known positive effects on human health $(12,13)$, there is little information about the effect of mushroom consumption in poults. However, on turkeys, it has been reported that $A$. bisporus, due to its polysaccharides content, has a prebiotic-like effect and improves the efficiency in digestibility and utilization of feed $(14,15)$.

The I ranian production of mushroom and truffles has increased from 10,000 t in 1997 to 82,000 in 2011 (FAO estimate), holding in this year the $8^{\text {th }}$ place in the world mushroom production(3). The mushroom industry produces significant quantities of mushroom wastage that are discarded during the mushroom production process. The recycling of this by-product as feed additive for broiler production should increase the efficiency of the Iranian food industry by reducing costs.

The positive impact of antibiotic as growth promoter (AGP) is well known in the poultry industry due to the suppression of harmful microorganisms(16) and to inhibition of inflammation effects(17). Association of bacterial resistance with the addition of AGP in the chicken feed may be questionable $(18,19)$ and it seems that is the precautionary principle the main reason for the banning of AGP in several countries $(20,21)$. In this sense, the use of 
antibióticos con la adición de AGP en el alimento para pollos puede ser cuestionable(18,19) y parece que es el principio de precaución la principal razón para la prohibición de estos en varios países $(20,21)$. En este sentido, el uso de antibióticos que no se utilizan en medicina humana y que todavía no causan resistencia podría ser una buena alternativa. Este es el caso del flavofosfolipol, un antimicrobiano fosfoglicolipídico producido por varias cepas de Streptomyces, que no se utiliza para el tratamiento de las infecciones humanas, por lo que todavía se puede agregar legalmente en dietas de aves de corral en muchos países(16).

Resultados previos de nuestro grupo han demostrado que la adición de harina de champiñón $(\mathrm{HC})$ y flavofosfolipol tienen un efecto beneficioso sobre el rendimiento en pollos de engorda, ya que incrementa la ganancia de peso, el índice de conversión y el peso corporal a los 42 días de crecimiento (Shamsi et al. comunicación personal). El objetivo de este estudio fue demostrar que estos efectos positivos sobre la productividad del pollo están directamente relacionados con el rendimiento de la canal. Adicionalmente se pretendía también evaluar el efecto sobre el peso de las vísceras y las medidas del tracto digestivo.

Este estudio se llevó a cabo en la granja de aves de corral de la Facultad de Agricultura de la Universidad Islámica Azad, Rasht Branch, en la ciudad de Ramsar, Irán ( $50^{\circ} 40^{\prime} \mathrm{N}$ y $36^{\circ} 54^{\prime}$ E) durante la temporada de verano (cálida), entre julio-septiembre de 2013, a lo largo de un total de 42 días. El experimento se aprobó por el Consejo Científico de la Universidad Islámica Azad, y se llevó a cabo de acuerdo con las Directrices Internacionales para la investigación con animales(22).

Trescientos (300) pollos de engorda machos Ross 308 de un día de edad(23) se asignaron aleatoriamente a 10 grupos de tratamientos con tres réplicas por tratamiento. Cada réplica consistió en 10 pollitos alojados en corrales de $1.2 \mathrm{~m}^{2}$. Antes de que los animales fueran antibiotics not used in humans therapy and that do not cause resistance could still be an alternative. This is the case of flavophospholipol, a phosphoglycolipid antimicrobial produced by several strains of Streptomyces that is not used for treatment of human infections, which is why it can still be legally added in poultry diets in many countries(16).

Previous results from our group have shown that the addition of dietary mushroom powder and flavophospholipol has a beneficial effect on growth performance as it showed positive on weight gain, feed conversion ratio and body weight at 42-d of growth (Shamsi et al. under review). The aim of this current study is to demonstrate that these previous positive effects on chicken performance are directly linked to the carcass yield. In addition, the effect on viscera weight and digestive tract measurement have also been evaluated.

This study was performed in the Poultry Farm Facilities in Ramsar city $\left(50^{\circ} 40^{\prime} \mathrm{N}\right.$ and $36^{\circ} 54^{\prime}$ E) and the Agriculture Faculty, Islamic Azad University, Rasht Branch, Iran, during the summer (warm) season, between JulySeptember 2013, for a total of $42 \mathrm{~d}$. The experiments were approved by the Scientific Board of the Islamic Azad University, and were conducted as per International Guidelines for research involving animals(22).

Three hundred (300) one-day-old male Ross 308 broilers (23) were randomly assigned to 10 treatments with three replicates per treatment. Each replicate consisted of 10 chicks housed in pens of $1.2 \mathrm{~m}^{2}$. Before the animals were placed in their pens, all the facilities were thoroughly cleaned and disinfected with 1:125 and 1:250 solution of Multifenelic. Other equipment including drinkers and feeders was disinfected with $20 \%$ Benzalkonium chloride - Germo Killer. All the windows and the ventilators were gasified with Formalex ${ }^{\circledR}$ solution.

The room temperature was adjusted to approximately $33 \stackrel{\circ}{ } \mathrm{C}$ on $\mathrm{d} 1$, and then was 
colocados en sus corrales, todas las instalaciones estaban completamente limpias y desinfectadas con 1:125 y 1:250 de solución multifenólica. Otros equipos, incluyendo bebederos y comederos, se desinfectaron con $20 \%$ de cloruro de benzalconio - Germo Killer. Todas las ventanas y los ventiladores se gasificaron con solución Formalex ${ }^{\circledR}$.

La temperatura ambiente se ajustó a aproximadamente a $33 \stackrel{\circ}{\circ}$ en el día 1 , y luego se redujo gradualmente a $24 \stackrel{\circ}{ } \mathrm{C}$. Los corrales se equiparon con calentadores eléctricos. La iluminación contaba con un programa de $23 \mathrm{~L}: 10$ (1900-2000 h). La humedad se mantuvo entre 55 y $65 \%$ en el periodo de crecimiento inicial, por pulverización de agua en el suelo. Los pollos se vacunaron contra la enfermedad de Newcastle y de Gumboro. Para reducir el estrés, a las $24 \mathrm{~h}$ después de la vacunación, se suministró un multi-vitamínico y solución electrolítica (1:1000) a través del agua potable.

El agua y el alimento se suministraron ad libitum durante todo el período experimental. Los requerimientos nutricionales se basaron en el catálogo para la crianza de la estirpe Ross(23). La alimentación se formuló para que fuera isoprotéica e isoenergética para todos los tratamientos. La composición de las dietas y su contenido nutricional se presentan en el Cuadro 1. Se utilizaron tres formulaciones de alimento durante el período de cría: el periodo de iniciación 1-14 días de edad (dde), crecimiento (15-28 dde) y de finalización (29-42 dde). El alimento sobrante en los comederos se pesó y se registró semanalmente.

Los champiñones (A. bispours) se obtuvieron de un productor local (Compañía Pars, Ramsar, I rán), se secaron a $60 \stackrel{\circ}{ } \mathrm{C}$, seguido de molienda, y se añadieron a las dietas experimentales. Los tratamientos se seleccionaron para determinar cinco niveles de harina de champiñón $(0,0.5$, $1.0,1.5$ y $2.0 \mathrm{~g} / \mathrm{kg})$, y dos niveles de flavofosfolipol (Teif Azmoon Pars, Co., Teherán, Irán), 0 o $5.0 \mathrm{mg} / \mathrm{kg}$. gradually decreased to $24 \stackrel{\circ}{ } \mathrm{C}$. The pens were fitted with electrical heaters. Lighting was provided with a program of 23L: $1 \mathrm{~N}$ (from 1900 to $2000 \mathrm{~h}$ ). Humidity was maintained between 55 and $65 \%$ in the early growing period, by

Cuadro 1. Ingredientes y análisis de nutrientes de dietas utilizadas durante los periodos de inicio (1-4 días de edad), crecimiento (15-28 días) y finalización (29-42 días)

Table 1. Feed ingredients and nutrient analysis of diets used during the starter (1-14 $d$ of age), grower (15-28 $d$ of age), and finisher periods (29-42 $\mathrm{d}$ of age)

\begin{tabular}{|c|c|c|c|}
\hline & $\begin{array}{l}\text { Starter } \\
\text { period }\end{array}$ & $\begin{array}{l}\text { Grower } \\
\text { period }\end{array}$ & $\begin{array}{c}\text { Finisher } \\
\text { period }\end{array}$ \\
\hline \multicolumn{4}{|l|}{ Feed ingredients ( $/ \mathrm{kg})$} \\
\hline Corn & 474 & 454 & 420 \\
\hline Soybean meal & 395 & 330 & 264 \\
\hline Wheat & 70 & 150 & 250 \\
\hline Soybean oil & 15 & 25 & 28 \\
\hline Са\%22P\%18 & 21.5 & 18 & 15.7 \\
\hline Sodium bicarbonate ( $\mathrm{NaHCO} 3)$ & 1 & 1.4 & 1.9 \\
\hline $\mathrm{CaCO} 3$ & 9 & 8.7 & 8.5 \\
\hline $\mathrm{NaCl}$ & 2.6 & 2.4 & 2 \\
\hline Mineral mixture 1 & 2.5 & 2.5 & 2.5 \\
\hline Vitamin mixture 2 & 2.5 & 2.5 & 2.5 \\
\hline DL-Methionine & 2.9 & 2.5 & 2.3 \\
\hline Threonine & 1 & 0.8 & 0.5 \\
\hline Lysine-hydro-chloride & 2.5 & 1.7 & 1.6 \\
\hline Avizyme multienzyme & 0.5 & 0.5 & 0.5 \\
\hline Total & 1000 & 1000 & 1000 \\
\hline \multicolumn{4}{|l|}{ Nutrient analysis } \\
\hline Metabolizable energy, kcal/kg & 2900 & 2950 & 3000 \\
\hline Crude protein, $\%$ & 22 & 20 & 18 \\
\hline Digestible protein $\mathrm{SID}^{3}, \%$ & 20 & 18 & 16 \\
\hline Calcium, \% & 1.05 & 0.9 & 0.85 \\
\hline Available phosphorus, \% & 0.5 & 0.45 & 0.42 \\
\hline Sodium, \% & 0.16 & 0.16 & 0.16 \\
\hline $\mathrm{DCAB}, \mathrm{mEq} / \mathrm{kg}$ & 240 & 230 & 210 \\
\hline Lysine, \% & 1.4 & 1.18 & 1.04 \\
\hline Lysine SID, \% & 1.28 & 1.08 & 0.95 \\
\hline Methionine, \% & 0.63 & 0.57 & 0.5 \\
\hline Methionine SID, \% & 0.6 & 0.55 & 0.48 \\
\hline Methionine +Cysteine, \% & 0.99 & 0.91 & 0.82 \\
\hline Methionine +Cysteine SID, \% & 0.9 & 0.83 & 0.75 \\
\hline Threonine, $\%$ & 0.88 & 0.85 & 0.74 \\
\hline Threonine SID, \% & 0.76 & 0.74 & 0.65 \\
\hline
\end{tabular}

1 Calcium Pantothenate: $4 \mathrm{mg} / \mathrm{g}$; Niacin: $15 \mathrm{mg} / \mathrm{g}$; Vitamin B6: $13 \mathrm{mg} / \mathrm{g}$; Cu: $3 \mathrm{mg} / \mathrm{g}$; Zn: $15 \mathrm{mg} / \mathrm{g}$; Mn: $20 \mathrm{mg} / \mathrm{g}$; Fe: 10 $\mathrm{mg} / \mathrm{g} ; \mathrm{K}: 0.3 \mathrm{mg} / \mathrm{g}$.

2 Vitamin A: $5000 \mathrm{IU} / \mathrm{g}$; Vitamin D3: $500 \mathrm{IU} / \mathrm{g}$; Vitamin E: 3 $\mathrm{mg} / \mathrm{g}$; Vitamin K3: $1.5 \mathrm{mg} / \mathrm{g}$; Vitamin B2: $1 \mathrm{mg} / \mathrm{g}$.

3 SID (Standardized Ileal Digestible). 
Al final del estudio, se seleccionó un ave por corral (un total de tres aves por tratamiento) basada en el peso promedio del grupo, sobre las que se obtuvo el peso corporal vivo, tras lo cual fueron sacrificadas y desangradas completamente. Se eliminó la cabeza, vísceras y patas. Las aves se diseccionaron para apartar y determinar el rendimiento de diferentes vísceras, tracto digestivo y partes de la canal. Se pesaron el buche vacío, proventrículo vacío, molleja vacía, grasa abdominal, timo, bazo, bolsa de Fabricio, hígado, riñones, páncreas, pulmones, corazón y cerebro. El intestino delgado vacío se dividió en duodeno (del inicio de la molleja al final de la curva del páncreas), yeyuno (desde la curva del páncreas al divertículo de Meckel) e íleon (del divertículo de Meckel a la unión íleointestino-ciego). El intestino grueso vacío se dividió en ciego derecho e izquierdo, colon y recto. Estas partes se pesaron y midieron, obteniéndose longitud, anchura y diámetro.

Los pesos relativos se calcularon como un porcentaje del peso vivo al sacrificio: la canal se dejó reposar durante una hora para eliminar el exceso de agua y posteriormente durante toda la noche en un refrigerador a $4 \pm 1 \stackrel{\circ}{ }$, y se utilizó la relación entre el peso eviscerado y el peso vivo x 100. Posteriormente la canal se diseccionó, obteniendo la pechuga, muslos, alas, cuello y notarium, los cuales se pesaron. Estas mediciones se utilizaron para calcular el porcentaje de estas partes del peso de la canal.

Modelos lineales generales (GLM, SPSS 15.0, Chicago, IL, EE.UU.) examinaron los efectos de HC y flavofosfolipol en la canal, pesos de vísceras y sobre las medidas del tracto digestivo, en un arreglo factorial $2 \times 5$ usando: $Y_{i j}=\grave{i}+A_{j}+B_{k}+A_{j} B_{k}+e_{i j k}$. Las variables que resultaron significativas con los GLM se analizaron con ANOVA de una vía para encontrar diferencias entre los tratamientos, (diferentes niveles de adición de $\mathrm{HC}$ o antibiótico vs grupos de adición de antibióticos). El test de Tukey se utilizó para determinar las diferencias entre los grupos. spraying water on the floor. The chicks were vaccinated against Newcastle and Gumboro diseases. Within $24 \mathrm{~h}$ after vaccination, a multiple-vitamin and electrolytic solution (1:1000) was offered via drinking water to reduce stress.

Water and feed were supplied ad libitum during the entire experimental period. The nutritional requirements were based on Ross strain rearing catalogue(23). The feed was formulated to be iso-proteic and iso-energetic for all treatments. Composition of the diets and their nutrient contents are presented in Table 1 . Three types of feed formulations were used during the rearing period: the starter period (1-14 $d$ of age (DOA), grower period (15-28 DOA) and the finisher period (29-42 DOA). Feed remaining in feeders was weighed and recorded weekly.

Mushrooms (A. bispours) were obtained from a local mushroom producer (Pars Company, Ramsar,

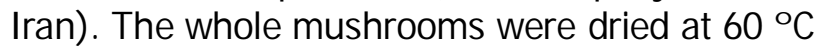
followed by grinding, and added to the experimental diets. Treatments were selected to determine five levels of mushroom powder (MP; i.e., $0,0.5,1.0,1.5$, and $2.0 \mathrm{~g} / \mathrm{kg}$ ), and two levels of antimicrobial flavophospholipol (Teif Azmoon Pars, Co., Tehran, Iran), 0 or 5.0 mg/kg.

At the end of the study, one bird per pen (totalling three birds per treatment) was selected based on the average weight of the group, weighed to obtain live body weight followed by killing and complete bleeding. Head, viscera and shanks were removed. The birds were dissected to remove and determine the yield of different viscera, digestive tract and carcass parts. Empty crop, empty proventriculus, empty gizzard, abdominal fat pad, thymus, spleen, bursa of Fabricius, liver, kidneys, pancreas, lungs, hearth and brain were weighed. Empty small intestine was divided into duodenum (from the gizzard outlet to the end of the pancreatic loop), jejunum (from the pancreatic loop to Meckel's diverticulum) and ileum (from Meckel's diverticulum to the ileo-caeco-colic junction) and these three different parts were weighted. 
El efecto de alimentar a los pollos con distintos niveles de harina de champiñón junto a la adición o no de flavofosfolipol sobre el peso canal, pechuga, muslos, alas, órganos comestibles, órganos relacionados con la inmunidad y las diferentes partes del tracto digestivo se muestran en los Cuadros 2 a 4 . El peso de la canal fría fue influido tanto por la inclusión del antibiótico como de la HC (Cuadro 2). Las mismas variables afectaron positivamente el peso de la canal eviscerada (Modelo $\mathrm{F}_{3.26}=$ 10.70, $\mathrm{P}<0.001, \mathrm{R}^{2}=56.8 \%$; coeficientes: $\mathrm{HC}=$ $7.4 \pm 36.2, P<0.001$, antibiótico $=212.4 \pm 62.6$, $\mathrm{P}<0.01$, interacción $=-196.7 \pm 51.1, \mathrm{P}<0.01$ ) . La inclusión de flavofosfolipol en la dieta mejoró algunas de las características de la canal: aves con mayor peso de pechuga, así como un mayor peso relativo de la misma (Modelo $\mathrm{F}_{3.26}=6.23$, $\mathrm{P}<0.05, \mathrm{R}^{2}=18.2 \%$; coeficiente: antibiótico=
Length, width and diameter of different parts were measured. Empty large intestine was divided into right and left cecum, colon and rectum. These parts were weighted and measured. Relative weights were calculated as a percentage of live weight at slaughter. Carcass was left for one hour to remove excess water and allowed to sit overnight in a refrigerator at $4 \pm 1 \stackrel{\circ}{ } \mathrm{C}$ using a ratio between the eviscerated weight and live weight $x$ 100. Carcass was then portioned; breast, drumsticks, wings, neck and notarium were obtained and weighed. Data from these measurements were used to calculate the percentage of part to the carcass weight.

General Lineal Models (GLM, SPSS 15.0, Chicago, IL, USA) examined the effects of MP and flavophospholipol on carcass and viscera weights

Cuadro 2. Efecto de flavofosfolipol (Ab), añadido a las dietas que contienen harina de champiñón (MP) sobre pesos de componentes de la canal $(\mathrm{g})$ de pollos de engorda los 42 días de edad

Table 2. Effect of flavophospholipol (Ab, 0.0 or $5.0 \mathrm{mg} / \mathrm{kg}$ ) added to diets containing mushroom powder (MP) at 0.0 , $0.5,1.0 ., 1.5$ or $2.0 \mathrm{~g} / \mathrm{kg}$ on different carcass weights $(\mathrm{g})$ of male broiler chicken at $42 \mathrm{~d}$ of age

\begin{tabular}{|c|c|c|c|c|c|c|c|c|c|}
\hline Treatment & Cold carcass weight & Dressing percentage & Abdominal fat & Breast & Drumsticks & Wings & Head & Neck & Notarium \\
\hline \multicolumn{10}{|c|}{ Antibiotic addition $\mathrm{x}$ mushroom powder level } \\
\hline MPO & $1958.3^{a}$ & 77.3 & 15.1 & $605.3^{a}$ & $532.3^{a}$ & 134.7 & 64.7 & $51.3^{a, b}$ & 55.3 \\
\hline MPOAb & $2314.7^{b}$ & 77.7 & 18.4 & $812.0^{b}$ & $631.3^{a}, \mathrm{~b}$ & 106.7 & 67.7 & $55.3 a, b$ & 59.3 \\
\hline MP0.5 & $2273.3^{a, b}$ & 78.2 & 24.0 & $804.7^{b}$ & $634.7^{a, b}$ & 109.3 & 69.3 & $52.0^{a, b}$ & 56.7 \\
\hline MP0.5Ab & $2281.3^{a, b}$ & 78.8 & 36.3 & $826.0^{b}$ & $632.0^{a, b}$ & 92.0 & 64.7 & $47.3^{a}$ & 58.0 \\
\hline MP1.0 & $2300.3 a, b$ & 79.0 & 28.1 & $763.7 a, b$ & $655.7 a, b$ & 114.7 & 68.7 & $54.7 a, b$ & 58.7 \\
\hline MP1.0Ab & $2426.3^{b}$ & 79.0 & 29.7 & $842.3^{a, b}$ & $674.0^{a, b}$ & 111.3 & 70.3 & $54.0^{a, b}$ & 59.3 \\
\hline MP1.5 & $2538.0^{b}$ & 78.4 & 23.7 & $904.7^{b}$ & $679.3^{b}$ & 109.3 & 74.3 & $62.0^{a}, \mathrm{~b}$ & 54.7 \\
\hline MP1.5Ab & $2258.0 \mathrm{a}, \mathrm{b}$ & 78.6 & 22.6 & $813.0^{b}$ & $622.3 \mathrm{a}, \mathrm{b}$ & 100.3 & 71.7 & $54.0 \mathrm{a}, \mathrm{b}$ & 60.0 \\
\hline MP2.0 & $2529.3^{b}$ & 77.6 & 38.2 & $832.7^{b}$ & $726.7^{b}$ & 111.3 & 76.0 & $64.0^{b}$ & 56.7 \\
\hline MP2.0Ab & $2346.0^{b}$ & 78.2 & 20.6 & $843.0^{b}$ & $617.3^{a, b}$ & 105.3 & 64.3 & $52.7^{a, b}$ & 60.0 \\
\hline Pooled SEM & 34.2 & 0.3 & 2.1 & 17.0 & 11.8 & 3.7 & 1.2 & 1.2 & 1.6 \\
\hline \multicolumn{10}{|l|}{ Main effect } \\
\hline 0.0 & $2319.9 a$ & 78.1 & 25.8 & $782.2^{\mathrm{a}}$ & $645.7^{a}$ & 115.9 & 70.6 & $56.8^{a}$ & 56.4 \\
\hline 5.0 & $2325.3^{a}$ & 78.5 & 25.5 & $827.3^{a}$ & $635.4^{\mathrm{a}}$ & 103.1 & 67.7 & $52.7^{a}$ & 59.3 \\
\hline \multicolumn{10}{|c|}{ Mushroom powder } \\
\hline 0.0 & $2136.5^{a}$ & 77.5 & 16.8 & $708.7^{a}$ & $581.8^{a}$ & 120.7 & 66.2 & $53.3^{a}$ & 57.3 \\
\hline 0.5 & $2277.3^{a, b}$ & 78.5 & 30.2 & $815.3^{a, b}$ & $633.3^{a}$ & 100.7 & 67.0 & $49.7^{a}$ & 57.3 \\
\hline 1.0 & $2363.3 a, b$ & 79.0 & 28.9 & $803.0^{a}, \mathrm{~b}$ & $664.8^{a}$ & 113.0 & 69.5 & $54.3^{a}$ & 59.0 \\
\hline 1.5 & $2398.0^{a, b}$ & 78.5 & 23.1 & $858.8^{b}$ & $650.8^{a}$ & 104.8 & 73.0 & $58.0^{a}$ & 57.3 \\
\hline 2.0 & $2437.7^{b}$ & 77.9 & 29.4 & $837.8^{a, b}$ & $672.0^{a}$ & 108.3 & 70.2 & $58.3^{a}$ & 58.3 \\
\hline \multicolumn{10}{|c|}{ Significance of ANOVA } \\
\hline Antibiotic & $<0.01$ & NS & NS & $<0.01$ & NS & NS & NS & NS & NS \\
\hline MP & $<0.001$ & NS & NS & $<0.001$ & $<0.05$ & NS & NS & $<0.05$ & NS \\
\hline$A b \times M P$ & $<0.001$ & NS & NS & $<0.01$ & NS & NS & NS & $<0.01$ & NS \\
\hline
\end{tabular}

ab Means with the same letter are not significant different $(P<0.05)$. 
$2.0 \pm 0.8, \mathrm{P}<0.05)$. En contraste, el flavofosfolipol mostró un efecto negativo sobre el peso relativo de las alas (Modelo $\mathrm{F}_{3.26}=3.79, \mathrm{P}<0.05, \mathrm{R}^{2}=$ $31.3 \%$; coeficientes: antibiótico $=-0.7 \pm 0.2$, $\mathrm{P}<0.01$, la interacción entre $\mathrm{HC}$ y antibiótico $=$ $0.03 \pm 01, P<0.05$ ) y el cuello (Modelo $F_{3.26}=$ 4.98, $P<0.05, R^{2}=15.1 \%$; coeficiente: antibiótico $=-0.2 \pm 0.1, P<0.05)$.

Las raciones de alimentación con $\mathrm{HC}$ mejoraron los pesos de pechuga, muslo y cuello (Cuadro 2). El efecto de las dos variables estudiadas sobre el peso de las distintas vísceras se muestra en el Cuadro 3. La inclusión de flavofosfolipol al $5.0 \%$ en la dieta no logró mejorar el peso de ningún órgano. Por otro lado, la harina de champiñón mostró un efecto positivo sobre el and on the measurements of the digestive tract in a $2 \times 5$ factorial arrangement using $Y_{i j_{k}}=i+A_{j}+B_{k}+A_{j} B_{k}+e_{i j k}$ statistical formula. Variables found significant with GLM were tested with one-way ANOVA to find differences between treatments, (different levels of MP addition or antibiotic vs no antibiotic addition groups). Tukey's test was used to determine disparities among the groups.

Cold carcass weight was affected by both the inclusion of the antibiotic and MP (Table 2). The same variables affected positively too the dressed carcass weight (Model $F_{3,26}=10.70$, $\mathrm{P}<0.001, \mathrm{R}^{2}=56.8 \%$; coefficients: $\mathrm{MP}=7.4 \pm$ 36.2, $P<0.001$, antibiotic $=212.4 \pm 62.6$, $\mathrm{P}<0.01$, interaction between both factors $=-196.7$

Cuadro 3. Efecto de flavofosfolipol (Ab) añadido a las dietas que contienen harina de champiñón (MP) en los pesos de las vísceras $(\mathrm{g})$ de pollos de engorda a los 42 días de edad

Table 3. Effect of flavophospholipol (Ab, 0.0 or $5.0 \mathrm{mg} / \mathrm{kg}$ ) added to diets containing mushroom powder (MP) at 0.0 , $0.5,1.0 ., 1.5$ or $2.0 \mathrm{~g} / \mathrm{kg}$ on viscera weights $(\mathrm{g})$ of male broiler chicken at $42 \mathrm{~d}$ of age

\begin{tabular}{|c|c|c|c|c|c|c|c|c|c|}
\hline Treatment & Thymus & Spleen & Bursa Fabricius & Liver & Kidneys & Pancreas & Lungs & Heart & Brain \\
\hline \multicolumn{10}{|c|}{ Antibiotic addition $\mathrm{x}$ mushroom powder level } \\
\hline MPO & $2.9 \mathrm{a}$ & $3.0^{\mathrm{a}}$ & $2.3^{a}$ & $50.6^{a}$ & 10.4 & 4.8 & 13.3 & 16.7 & $1.9^{a}$ \\
\hline MPOAb & $5.2^{a, b}$ & $2.7^{a}$ & $2.3^{a}$ & $51.8^{a}$ & 12.3 & 5.9 & 12.6 & 15.4 & $1.7^{\mathrm{a}}$ \\
\hline MP0.5 & $5.3^{a}, \mathrm{~b}$ & $2.4^{a}$ & $2.0^{a}$ & $49.7^{a}$ & 10.5 & 5.7 & 12.8 & 14.6 & $2.4^{a}$ \\
\hline MP0.5Ab & $4.7^{a, b}$ & $2.2^{a}$ & $3.8^{b}$ & $50.3^{a}$ & 14.4 & 6.5 & 12.9 & 12.7 & $2.3^{a}$ \\
\hline MP1.0 & $4.6^{a, b}$ & $2.0^{a}$ & $4.6^{b}$ & $57.9^{a}$ & 12.7 & 5.9 & 13.8 & 14.5 & $2.9 \mathrm{a}$ \\
\hline MP1.0Ab & $5.7^{b}$ & $2.7^{a}$ & $4.3^{b}$ & $49.7^{a}$ & 12.4 & 6.2 & 13.0 & 14.7 & $2.5^{a}$ \\
\hline MP1.5 & $5.2^{a, b}$ & $2.7^{a}$ & $4.5^{b}$ & $63.2^{\mathrm{a}}$ & 13.3 & 5.7 & 15.1 & 14.1 & $2.5^{\mathrm{a}}$ \\
\hline MP1.5Ab & $6.3^{b}$ & $3.1^{a}$ & $4.6^{b}$ & $50.2^{a}$ & 11.2 & 5.5 & 14.2 & 16.4 & $2.9^{a}$ \\
\hline MP2.0 & $6.4^{b}$ & $3.6 \mathrm{a}$ & $4.4^{b}$ & $56.3^{a}$ & 12.3 & 6.4 & 11.5 & 12.2 & $2.8^{a}$ \\
\hline MP2.0Ab & $5.8^{b}$ & $3.4^{a}$ & $4.5^{b}$ & $57.1^{a}$ & 12.9 & 6.0 & 15.6 & 18.4 & $2.8^{a}$ \\
\hline Pooled SEM & 0.2 & 0.1 & 0.2 & 1.1 & 0.1 & 0.2 & 0.5 & 0.6 & 0.1 \\
\hline \multicolumn{10}{|l|}{ Main effect } \\
\hline \multicolumn{10}{|l|}{ Antibiotic } \\
\hline 0.0 & $4.9 a$ & $2.7^{a}$ & $3.6^{a}$ & $55.5^{a}$ & 11.8 & 5.7 & 13.3 & 14.4 & $2.5^{a}$ \\
\hline 5.0 & $5.5^{a}$ & $2.8^{a}$ & $3.9 a$ & $51.8^{a}$ & 12.6 & 8.0 & 13.7 & 15.5 & $2.4^{a}$ \\
\hline \multicolumn{10}{|c|}{ Mushroom powder } \\
\hline 0.0 & $4.1^{\mathrm{a}}$ & $2.9 a, b$ & $2.3^{a}$ & $51.2^{a}$ & 11.3 & 5.2 & 13.0 & 15.0 & $1.8^{a}$ \\
\hline 0.5 & $5.0^{a, b}$ & $2.3^{a}$ & $2.9^{a}$ & $50.0^{a}$ & 12.5 & 6.1 & 12.8 & 13.6 & $2.4^{a, b}$ \\
\hline 1.0 & $5.2^{a, b}$ & $2.3^{a}$ & $4.4^{b}$ & $53.8^{a}$ & 12.5 & 6.1 & 13.4 & 14.6 & $2.7^{b}$ \\
\hline 1.5 & $5.8^{a, b}$ & $2.9 a, b$ & $4.6^{b}$ & $56.7^{b}$ & 12.2 & 5.6 & 14.7 & 15.2 & $2.7^{b}$ \\
\hline 2.0 & $6.1^{\mathrm{b}}$ & $3.5^{b}$ & $4.5^{b}$ & $56.7^{b}$ & 12.6 & 6.2 & 13.5 & 15.3 & $2.8^{b}$ \\
\hline \multicolumn{10}{|c|}{ Significance of ANOVA } \\
\hline Antibiotic & NS & NS & NS & NS & NS & NS & NS & NS & NS \\
\hline MP & $<0.01$ & $<0.05$ & $<0.001$ & $<0.01$ & NS & NS & NS & NS & $<0.01$ \\
\hline$A b \times M P$ & NS & NS & NS & $<0.05$ & NS & NS & NS & NS & NS \\
\hline
\end{tabular}

ab Means with the same letter are not significant different $(P<0.05)$. 
peso del timo, bazo, bolsa de Fabricio, hígado y cerebro. Por último, los efectos de la adición a la dieta del antibiótico y la $\mathrm{HC}$ en las medidas gastrointestinales se muestran en los Cuadros 4.1, 4.2 y 4.3. No hubo efecto del antibiótico en ninguna de las mediciones gastrointestinales tomadas y la HC mostró diferencias estadísticamente significativas en sólo 6 de los 27 rasgos medidos.

Los resultados del presente estudio muestran un efecto positivo del antibiótico y la $\mathrm{HC}$ en el peso de la canal en frío. Aunque la adición de flavophopholipol mostró un efecto negativo en el peso relativo del cuello y las alas, tuvo un $\pm 51.1, \mathrm{P}<0.01$ ). Inclusion of flavophospholipol in diet improved some of the measured carcass traits. In this sense, the addition of the antibiotic resulted in birds with greater breast weight as well as greater relative breast weight (Model $F_{3,26}=6.23, P<0.05, R^{2}=18.2 \%$; coefficient: antibiotic $=2.0 \pm 0.8, P<0.05)$. In contrast, dietary flavophospholipol showed a negative effect relative weight of wings (Model $\mathrm{F}_{3,26}=$ 3.79, $P<0.05, R^{2}=31.3 \%$; coefficients: antibiotic $=-0.7 \pm 0.2, P<0.01$, interaction between MP and antibiotic $=0.03 \pm 0.1, P<0.05$ ) and neck (Model $\mathrm{F}_{3,26}=4.98, \mathrm{P}<0.05, \mathrm{R}^{2}=$ $15.1 \%$; coefficient: antibiotic $=-0.2 \pm 0.1$, $\mathrm{P}<0.05)$.

Cuadro 4.1. Efecto de flavofosfolipol (Ab) añadido a dietas que contienen harina de champiñón (MP) en el peso (g) y medidas $(\mathrm{mm})$ del sistema digestivo de pollos de engorda machos a los 42 días de edad

Table 4.1. Effect of flavophospholipol (Ab, 0.0 or $5.0 \mathrm{mg} / \mathrm{kg}$ ) added to diets containing mushroom powder (MP) at $0.0,0.5,1.0,1.5$ or $2.0 \mathrm{~g} / \mathrm{kg}$ on digestive system (weights, $\mathrm{g}$ and measurements, $\mathrm{mm}$ ) of male broiler chicken at $42 \mathrm{~d}$ of age

\begin{tabular}{|c|c|c|c|c|c|c|c|c|c|}
\hline \multirow[b]{2}{*}{ Treatment } & \multirow{2}{*}{$\begin{array}{c}\text { Crop } \\
\text { weight }\end{array}$} & \multicolumn{4}{|c|}{ Duodenum } & \multicolumn{4}{|c|}{ Jejunum } \\
\hline & & Weight & Length & Width & Diameter & Weight & Length & Width & Diameter \\
\hline \multicolumn{10}{|c|}{ Antibiotic addition $\mathrm{x}$ mushroom powder level } \\
\hline MPO & 11.0 & 18.5 & 390.0 & 5.4 & 0.26 & $45.4^{\mathrm{a}}$ & 670.0 & 6.4 & 0.34 \\
\hline MPOAb & 8.2 & 18.3 & 368.3 & 5.4 & 0.25 & $41.0^{a}$ & 640.0 & 6.6 & 0.21 \\
\hline MP0.5 & 5.4 & 18.5 & 391.7 & 6.1 & 0.17 & $49.9^{a}$ & 736.7 & 7.7 & 0.33 \\
\hline MP0.5Ab & 10.5 & 18.4 & 408.3 & 5.5 & 0.23 & $42.2^{a}$ & 696.7 & 6.8 & 0.26 \\
\hline MP1.0 & 8.0 & 16.3 & 371.7 & 6.7 & 0.33 & $46.3^{a}$ & 683.3 & 8.3 & 0.30 \\
\hline MP1.0Ab & 14.6 & 17.6 & 400.0 & 5.4 & 0.28 & $54.4^{a}$ & 790.0 & 7.2 & 0.40 \\
\hline MP1.5 & 12.7 & 18.7 & 350.0 & 5.3 & 0.40 & $55.7^{a}$ & 736.7 & 7.5 & 0.43 \\
\hline MP1.5Ab & 5.7 & 15.3 & 333.3 & 4.9 & 0.34 & $44.1^{\mathrm{a}}$ & 700.0 & 8.0 & 0.28 \\
\hline MP2.0 & 14.0 & 23.3 & 401.7 & 6.5 & 0.27 & $60.1^{a}$ & 793.3 & 7.9 & 0.30 \\
\hline MP2.0Ab & 8.5 & 17.1 & 375.0 & 5.7 & 0.33 & $48.8^{a}$ & 693.3 & 6.4 & 0.40 \\
\hline Pooled SEM & 1.0 & 0.6 & 7.0 & 0.2 & 0.02 & 1.7 & 15.3 & 0.2 & 0.02 \\
\hline \multicolumn{10}{|l|}{ Main effect } \\
\hline \multicolumn{10}{|l|}{ Antibiotic } \\
\hline 0.0 & 10.2 & 19.1 & 381.0 & 6.0 & 0.29 & $51.5^{a}$ & 724.0 & 7.6 & 0.34 \\
\hline 5.0 & 9.5 & 17.4 & 377.0 & 5.4 & 0.29 & $46.1^{a}$ & 704.0 & 7.0 & 0.31 \\
\hline \multicolumn{10}{|c|}{ Mushroom powder } \\
\hline 0.0 & 9.6 & 18.4 & 379.2 & 5.4 & 0.26 & $43.2^{a}$ & 655.0 & 6.5 & 0.28 \\
\hline 0.5 & 8.0 & 18.5 & 400.0 & 5.8 & 0.20 & $46.1^{\mathrm{a}}$ & 716.7 & 7.3 & 0.30 \\
\hline 1.0 & 11.3 & 17.0 & 385.8 & 6.0 & 0.31 & $50.3^{a}$ & 736.7 & 7.8 & 0.35 \\
\hline 1.5 & 9.2 & 17.0 & 341.7 & 5.1 & 0.38 & $49.9^{a}$ & 718.3 & 7.8 & 0.36 \\
\hline 2.0 & 11.3 & 20.2 & 388.3 & 6.1 & 0.31 & $54.5^{a}$ & 718.3 & 7.1 & 0.35 \\
\hline \multicolumn{10}{|c|}{ Significance of ANOVA } \\
\hline Antibiotic & NS & NS & NS & NS & NS & NS & NS & NS & NS \\
\hline MP & NS & NS & NS & NS & NS & $<0.05$ & NS & NS & NS \\
\hline$A b \times M P$ & NS & NS & NS & NS & NS & NS & NS & NS & NS \\
\hline
\end{tabular}

Means with the same letter are not significant different $(P<0.05)$. 
efecto positivo sobre el peso de la canal eviscerada. Por otro lado, la adición de HC tuvo un efecto positivo en el peso de partes de la canal que son económicamente importantes, como la pechuga y los muslos; sin embargo la combinación del antibiótico y la HC no ejercieron un efecto acumulativo en la canal a pesar de sus efectos individuales.

El mayor peso de los órganos inmunológicos (timo, bazo y bursa) al añadir HC sugiere un mejor estado inmune de los pollos, ya que el peso de los órganos linfoides refleja la capacidad del cuerpo para proporcionar linfocitos durante una respuesta inmune. En este sentido, las aves
Feeding rations containing MP yielded the highest breast, drumstick and neck weights (Table 2). The effect of the two variables studied on carcass viscera weights are shown in Table 3. Inclusion of flavophospholipol in diet at $5.0 \%$ failed to improve the weight of any organ weighed. On the other hand, mushroom powder showed a positive effect on the weight of thymus, spleen, bursa of Fabricius, liver and brain. Finally, the effects of the antibiotic and MP diet addition on the gastrointestinal traits are showed in Tables 4.1, 4.2 and 4.3. There was no effect of the antibiotic on any of the gastrointestinal measurements taken and MP only showed

Cuadro 4.2. Efecto de flavofosfolipol (Ab) añadido a dietas que contienen harina de champiñón (MP) en los pesos (g) y medidas $(\mathrm{mm})$ del sistema digestivo de pollos de engorda machos a los 42 días de edad

Table 4.2. Effect of flavophospholipol (Ab, 0.0 or $5.0 \mathrm{mg} / \mathrm{kg}$ ) added to diets containing mushroom powder (MP) at 0.0 , $0.5,1.0,1.5$ or $2.0 \mathrm{~g} / \mathrm{kg}$ on digestive system (weights, $\mathrm{g}$ and measurements, $\mathrm{mm}$ ) of male broiler chicken at $42 \mathrm{~d}$ of age

\begin{tabular}{|c|c|c|c|c|c|c|c|c|c|}
\hline \multirow[b]{2}{*}{ Treatment } & \multirow{2}{*}{$\begin{array}{l}\text { Proventriculus } \\
\text { weight }\end{array}$} & \multicolumn{4}{|c|}{ Ileum } & \multicolumn{4}{|c|}{ Colon } \\
\hline & & Weight & Length & Width & Diameter & Weight & Length & Width & Diameter \\
\hline \multicolumn{10}{|c|}{ Antibiotic addition $x$ mushroom powder level } \\
\hline MPO & 6.5 & 42.9 & 803.3 & $4.8^{a}$ & 0.27 & $2.7 \mathrm{a}$ & $81.7^{a}$ & 4.9 & 0.37 \\
\hline MPOAb & 6.7 & 42.6 & 726.7 & $5.7^{a}$ & 0.26 & $2.7^{a}$ & $76.7^{a}$ & 4.7 & 0.35 \\
\hline MP0.5 & 6.5 & 43.4 & 843.3 & $5.8^{a}$ & 0.30 & $3.3^{a}$ & $90.0^{a}$ & 7.6 & 0.41 \\
\hline MP0.5Ab & 8.0 & 41.7 & 730.0 & $7.0^{\mathrm{a}}$ & 0.36 & $2.9^{a}$ & $86.7^{a}$ & 6.4 & 0.35 \\
\hline MP1.0 & 6.1 & 36.2 & 760.0 & $6.5^{a}$ & 0.29 & $3.2^{\mathrm{a}}$ & $87.0^{a}$ & 5.6 & 0.57 \\
\hline MP1.0Ab & 6.7 & 51.5 & 886.7 & $7.2^{\mathrm{a}}$ & 0.37 & $3.8^{\mathrm{a}}$ & $91.7^{a}$ & 6.9 & 0.27 \\
\hline MP1.5 & 6.8 & 43.0 & 810.0 & $6.9^{a}$ & 0.32 & $3.8^{a}$ & $90.0^{a}$ & 5.9 & 0.37 \\
\hline MP1.5Ab & 6.2 & 32.0 & 753.3 & $6.8^{a}$ & 0.32 & $3.1^{\mathrm{a}}$ & $83.3^{a}$ & 6.1 & 0.38 \\
\hline MP2.0 & 7.1 & 54.8 & 926.7 & $7.5^{a}$ & 0.27 & $4.2^{\mathrm{a}}$ & $90.0^{a}$ & 6.9 & 0.44 \\
\hline MP2.0Ab & 7.5 & 44.4 & 825.0 & $7.2^{\mathrm{a}}$ & 0.35 & $3.5^{a}$ & $89.3^{a}$ & 6.3 & 0.39 \\
\hline Pooled SEM & 0.2 & 1.9 & 17.2 & 0.3 & 0.02 & 0.2 & 1.2 & 0.3 & 0.02 \\
\hline \multicolumn{10}{|l|}{ Main effect } \\
\hline \multicolumn{10}{|l|}{ Antibiotic } \\
\hline 0.0 & 6.6 & 44.1 & 828.7 & $6.3^{a}$ & 0.29 & $3.4^{\mathrm{a}}$ & $87.7^{a}$ & 6.2 & 0.43 \\
\hline 5.0 & 7.0 & 42.5 & 784.3 & $6.8^{a}$ & 0.33 & $3.2^{a}$ & $85.5^{a}$ & 6.1 & 0.35 \\
\hline \multicolumn{10}{|c|}{ Mushroom powder } \\
\hline 0.0 & 6.6 & 42.8 & 765.0 & $5.3^{a}$ & 0.26 & $2.7^{a}$ & $79.2^{a}$ & 4.8 & 0.36 \\
\hline 0.5 & 7.3 & 42.6 & 786.7 & $6.4^{\mathrm{a}}$ & 0.33 & $3.1^{a}$ & $88.3^{a, b}$ & 7.0 & 0.38 \\
\hline 1.0 & 6.4 & 43.9 & 823.3 & $6.6^{a}$ & 0.33 & $3.5^{\mathrm{a}}$ & $89.3^{b}$ & 6.2 & 0.42 \\
\hline 1.5 & 6.5 & 37.5 & 781.7 & $6.8^{a}$ & 0.32 & $3.4^{\mathrm{a}}$ & $86.7^{a, b}$ & 6.0 & 0.38 \\
\hline 2.0 & 7.3 & 49.6 & 875.8 & $7.3^{a}$ & 0.31 & $3.8^{a}$ & $89.7^{b}$ & 6.6 & 0.41 \\
\hline \multicolumn{10}{|c|}{ Significance of ANOVA } \\
\hline Antibiotic & NS & NS & NS & NS & NS & NS & NS & NS & NS \\
\hline MP & NS & NS & NS & $<0.05$ & NS & $<0.05$ & $<0.05$ & NS & NS \\
\hline$A b \times M P$ & NS & NS & NS & NS & NS & NS & NS & NS & NS \\
\hline
\end{tabular}

Means with the same letter are not significant different $(P<0.05)$. 
inmunodeprimidas o estresadas suelen tener órganos linfoides más pequeños, mientras que el mayor peso de la bursa refleja un mejor estado de salud. La bolsa de Fabricio tiene un papel extremadamente importante en la integridad morfológica y funcional del sistema inmune. El peso de la bursa en los pollos de engorda también refleja la respuesta anatómica a la alteración del sistema inmune debido al estrés(24). En concordancia con nuestros resultados, otros autores también han mostrado beneficios ligados a la inmunología en pollos de engorda alimentados con diferentes suplementos de hongos(25). Puesto que la composición de los mismos es compleja e incluye diferentes componentes, tales como statistically significance differences in 6 out of the 27 traits measured.

The results of the present study show that the effect of the antibiotic and MP are positive in the cold carcass weight. Although flavophopholipol had a negative effect on relative weights of neck and wings, however it had a positive effect on the eviscerated carcass weight. On the other hand, MP addition has a positive effect on carcass weight components that are economically important, e.g., breast and drumsticks. The combination of antibiotic and MP did not exert a cumulative effect on these carcass traits despite the effects shown as individual factors.

Cuadro 4.3. Efecto de flavofosfolipol (Ab) añadido a dietas que contienen harina de champiñón (MP) en los pesos (g) y medidas $(\mathrm{mm})$ del sistema digestivo de pollos de engorda machos a los 42 días de edad

Table 4.3. Effect of flavophospholipol (Ab, 0.0 or $5.0 \mathrm{mg} / \mathrm{kg}$ ) added to diets containing mushroom powder (MP) at 0.0, $0.5,1.0,1.5$ or $2.0 \mathrm{~g} / \mathrm{kg}$ on digestive system (weights, $\mathrm{g}$ and measurements, $\mathrm{mm}$ ) of male broiler chicken at $42 \mathrm{~d}$ of age

\begin{tabular}{|c|c|c|c|c|c|c|c|c|c|}
\hline \multirow[b]{2}{*}{ Treatment } & \multirow{2}{*}{$\begin{array}{l}\text { Gizzard } \\
\text { weight }\end{array}$} & \multicolumn{4}{|c|}{ Right cecum } & \multicolumn{4}{|c|}{ Left cecum } \\
\hline & & Weight & Length & Width & Diameter & Weight & Length & Width & Diameter \\
\hline \multicolumn{10}{|c|}{ Antibiotic addition $\mathrm{x}$ mushroom level } \\
\hline MP0 & 43.4 & 11.7 & 221.7 & 11.4 & $0.16^{\mathrm{a}}$ & $10.6^{a}$ & 218.3 & 13.8 & 0.20 \\
\hline MPOAb & 40.7 & 10.4 & 198.3 & 11.6 & $0.22^{\mathrm{a}}$ & $10.6^{a}$ & 191.7 & 11.1 & 0.24 \\
\hline MP0.5 & 44.1 & 8.1 & 181.3 & 9.5 & $0.20^{\mathrm{a}}$ & $7.9^{a}$ & 211.7 & 9.8 & 0.19 \\
\hline MP0.5Ab & 47.3 & 8.0 & 195.0 & 8.5 & $0.19^{a}$ & $7.4^{\mathrm{a}}$ & 195.0 & 8.6 & 0.28 \\
\hline MP1.0 & 37.5 & 6.8 & 181.7 & 11.7 & $0.20^{\mathrm{a}}$ & $7.2^{\mathrm{a}}$ & 183.3 & 9.8 & 0.28 \\
\hline MP1.0Ab & 53.2 & 7.7 & 208.3 & 10.5 & $0.28^{a}$ & $8.1^{\mathrm{a}}$ & 226.7 & 10.4 & 0.17 \\
\hline MP1.5 & 54.4 & 8.5 & 186.7 & 12.2 & $0.26^{a}$ & $8.0^{\mathrm{a}}$ & 191.7 & 10.1 & 0.20 \\
\hline MP1.5Ab & 44.3 & 7.2 & 183.3 & 10.4 & $0.25^{a}$ & $6.5^{\mathrm{a}}$ & 163.3 & 12.0 & 0.22 \\
\hline MP2.0 & 53.7 & 9.2 & 215.0 & 11.9 & $0.27^{a}$ & $8.3^{a}$ & 218.3 & 12.3 & 0.22 \\
\hline MP2.0Ab & 43.4 & 7.2 & 175.0 & 11.8 & $0.38^{a}$ & $6.7^{a}$ & 178.3 & 10.5 & 0.35 \\
\hline Pooled SEM & 1.3 & 0.5 & 3.8 & 0.6 & 0.02 & 0.4 & 4.6 & 0.6 & 0.02 \\
\hline \multicolumn{10}{|l|}{ Main effect } \\
\hline \multicolumn{10}{|l|}{ Antibiotic } \\
\hline 0.0 & 46.6 & 8.9 & 197.3 & 11.3 & $0.29 \mathrm{a}$ & $8.4^{\mathrm{a}}$ & 204.7 & 11.2 & 0.21 \\
\hline 5.0 & 45.8 & 8.1 & 192.0 & 10.6 & $0.28^{a}$ & $7.9 \mathrm{a}$ & 191.0 & 10.5 & 0.25 \\
\hline \multicolumn{10}{|c|}{ Mushroom powder } \\
\hline 0.0 & 42.1 & 11.0 & 210.0 & 11.5 & $0.19 a$ & $10.6^{\mathrm{a}}$ & 205.0 & 12.4 & 0.22 \\
\hline 0.5 & 45.7 & 8.1 & 188.2 & 9.0 & $0.25^{\mathrm{a}}$ & $7.7^{\mathrm{a}}$ & 203.3 & 9.2 & 0.24 \\
\hline 1.0 & 45.4 & 7.3 & 195.0 & 11.1 & $0.24^{a}$ & $7.6^{\mathrm{a}}$ & 205.1 & 10.1 & 0.20 \\
\hline 1.5 & 49.3 & 7.8 & 185.0 & 11.3 & $0.26^{a}$ & $7.2^{\mathrm{a}}$ & 177.5 & 11.1 & 0.21 \\
\hline 2.0 & 48.6 & 8.2 & 195.0 & 11.8 & $0.33^{a}$ & $7.5^{\mathrm{a}}$ & 198.3 & 11.4 & 0.29 \\
\hline \multicolumn{10}{|c|}{ Significance of ANOVA } \\
\hline Antibiotic & NS & NS & NS & NS & NS & NS & NS & NS & NS \\
\hline $\mathrm{MP}$ & NS & NS & NS & NS & $<0.05$ & $<0.05$ & NS & NS & NS \\
\hline$A b \times M P$ & NS & NS & NS & NS & NS & NS & NS & NS & NS \\
\hline
\end{tabular}

ab Means with the same letter are not significant different $(P<0.05)$ 
proteínas inmunomoduladoras (FIPS, por sus siglas en inglés)(26), los hongos comestibles no deben ser considerados sólo como un simple alimento.

Así, la actividad inmunoestimulante de A. bisporus ha sido plenamente demostrada; sus extractos de polisacáridos tienen un efecto inmunoestimulante induciendo la síntesis de IFN- $\delta$, que altera la transcripción de hasta 30 genes que a su vez producen variedad de respuestas fisiológicas y celulares(27). Además, A. bisporus es también una buena fuente de selenio(28), que se ha demostrado que mejora la actividad inmune humoral(29).

El mayor peso del hígado de los pollos tratados con $\mathrm{HC}$ podría reflejar el efecto antioxidante del champiñón común $(7,8,9)$. En este sentido, se ha observado que la administración de extracto etanólico de A. bisporus mejora de manera significativa la actividad de enzimas antioxidantes en hígado de ratones(25).

En términos generales, la suplementación de $\mathrm{HC}$ mostró un efecto muy débil en las diferentes medidas del tracto gastrointestinal. Se esperaban mayores diferencias, ya que se ha demostrado que los hongos afectan la morfología intestinal. Algunos investigadores han encontrado que el consumo de hongos modifica la conformación de la mucosa intestinal produciendo una mayor altura de las vellosidades en duodeno, yeyuno e íleon, cambios que afectan la capacidad de las aves para absorber nutrientes de la alimentación(15). Más sorprendente es el hecho de que no se encontró ningún efecto positivo al incluir flavofosfolipol, ya que el uso de AGP provoca cambios en la estructura del sistema gastrointestinal; la mayoría de los cuales son el resultado de la reducción en el peso total del intestino delgado debido más a los cambios en el espesor de la pared intestinal, que a cambios en su longitud(30,31,32). Algunos autores han indicado que la inclusión en la dieta de antibióticos, como promotores del crecimiento, reduce el peso del intestino por el adelgazamiento de la pared intestinal(33) y otros han encontrado un
Greater weight of the immune organs (thymus, spleen and bursa) with MP suggests a better immune status of the MP treatment chicks since lymphoid organ weights reflect the body's ability to provide lymphoid cells during an immune response. Thus, immunosuppressed or stressed birds usually have smaller lymphoid organs while higher bursa weight reflects better health status. The bursa of Fabricius has an extremely vital role in morphologic and functional integrity of the immune system. The weight of the bursa in broilers also reflects the anatomical response to immune system alteration due to stress(24). In accordance with our results, other authors have also reported immune-enhancing benefits in broiler chickens fed different mushroom supplements(25). Since the composition of mushrooms is complex and includes different proteins, such as fungal immunomodulating proteins (FIPs)(26), edible mushrooms should not be considered as only a simple food.

The immunostimulant activity of $A$ bisporus has been largely demonstrated; their polysaccharide extracts express an immunostimulating effect and induce synthesis of IFN- $\delta$, which alters transcription of up to 30 genes producing a variety of physiological and cellular responses(27). Furthermore, A. bisporus is also a good source of selenium(28), which has been shown to improve humoral immune activity(29).

The greater liver weight of chicks treated with mushroom extract could reflect the antioxidant effect of white button mushrooms $(7,8,9)$. It has been observed that the administration of ethanoic extract of A. bisporus significantly enhanced the activities of antioxidant enzymes in liver of mice(25).

Broadly speaking, supplementation of MP showed a very weak effect on the different measurements of the gastrointestinal tract. It was expected greater differences since it has been showed that the mushrooms affect intestinal morphology. Thus, researchers have found that mushroom intake influences mucosal architecture resulting in greater duodenal, 
menor peso del intestino delgado y el acortamiento del intestino en pavitos tratados con virginiamicina(34). Una explicación plausible es que los efectos negativos de AGP y los efectos positivos de los componentes de A. bisporus podrían estar traslapados. En cualquier caso, se requiere más investigación para llegar a una conclusión definitiva.

El uso de flavofosfolipol y $\mathrm{HC}$ tuvo efectos positivos en el crecimiento de los polluelos. Estos efectos se tradujeron en mayores pesos de partes de la canal con relevancia económica, como la pechuga y muslos. Los resultados muestran también un incremento general en los órganos relacionados con la inmunidad, lo cual refleja un mejor estado inmunológico de los pollos alimentados con harina de champiñón. Un incremento en el peso del hígado también se asoció con propiedades antioxidantes del champiñón común. Por último, en cuanto al efecto sobre los parámetros gastrointestinales, a pesar de la débil influencia observada, es necesaria una posterior investigación para una conclusión definitiva.

\section{LITERATURA CITADA}

1. Steinfeld H. Livestock production in the Asia and Pacific region-current status, issues and trends. World Anim Rev 1998; (90): 14-21.

2. Shariatmadari F. Poultry production and the industry in Iran. World Poultry Sci 2000; (56):55-65.

3. FAOSTAT. FAO Statistics. Food and Agriculture Organization of the United Nations 2014.

4. Tian $\mathrm{Y}$, Zeng $\mathrm{H}, \mathrm{Xu} Z$, Zheng B, Lin Y, Gan C, et al. Ultrasonicassisted extraction and antioxidant activity of polysaccharides recovered from white button mushroom (Agaricus bisporus). Carbohydr Polym 2012;88(2):522-529.

5. Chen S, Oh SR, Phung S, Hur G, Ye JJ, Kwok SL, et al. Anti-aromatase activity of phytochemicals in white button mushrooms (Agaricus bisporus). Cancer Res 2006; 66(24): 12026-12034.

6. Kohno K, Miyake M, Sano O, Tanaka-Kataoka M, Yamamoto $S$, Koya-Miyata $S$, et al. Anti-inflammatory and immunomodulatory properties of 2-Amino-3Hphenoxazin- 3one. Biol Pharma Bull 2008;31(10):1938-1945.

7. Tsai SY, Huang SJ, Lo SH, Wu TP, Lian PY, Mau JL. Flavour components and antioxidant properties of several cultivated mushrooms. Food Chem 2009;113(2):578-584. jejunal and ileal villus height, changes that affect the capacity of the birds to absorb nutrients from the feed(15). More surprising is the fact that it was not find any positive effect of including flavophospholipol, as AGP cause changes in the gross structure of the gastrointestinal system. Most of these changes are the result of reduction in overall weight of the small intestine due more to changes in the thickness of the intestinal wall rather than changes in the intestinal length $(30,31,32)$. Some authors have indicated that dietary inclusion of antibiotics, given as growth promoters, reduces intestine weight by thinning the intestinal wall(33) and some have found lower small intestine weight and shortening of the gut in poults treated with virginiamycin(34). A plausible explanation is that the negative effects of an AGP and the positive effects of the components of the A. bisporus could be overlapping. Further research is needed to arrive at a definitive conclusion.

In conclusion, the use of flavophospholipol and MP had a positive effect on the growth performance of chicks. These effects were translated into greater carcass components with economic relevance such as the breast and drumsticks. Results also show an overall increment in the immune related organs which reflects a better immune status of the broilers fed MP. An increment in the liver weight was also associated with antioxidative properties of the white mushroom button. Finally, regarding the effect on gastrointestinal parameters, despite the weak influence observed, further research is needed for a definitive conclusion.

\section{End of english version}

8. Jeong SC, Jeong YT, Yang BK, Islam R, Koyyalamudi SR, Pang G, et al. White button mushroom (Agaricus bispours) lowers blood glucose and cholesterol levels in diabetic and hypercholesterolemic rats. Nutr Res 2010;(30):49-56.

9. Kozarski M, Klaus A, Niksic M, Jakovljevic D, Helsper JP, Van Griensven LJ. Antioxidative and immunomodulating activities of polysaccharide extracts of the medicinal mushrooms Agaricus bisporus, Agaricus brasiliensis, 


\section{EFECTO DEL CHAMPIÑÓN Y FLAVOFOSFOLIPOL SOBRE LA CANAL EN POLLOS DE ENGORDA}

Ganoderma lucidum and Phellinus linteus. Food Chem 2011; 129(4): 1667-1675.

10. Moro C, Palacios I, Lozano M, D’Arrigo M, Guillamón E, Villares $A$, et al. Anti-inflammatory activity of methanolic extracts from edible mushrooms in LPS activated RAW 264.7 macrophages. Food Chem 2012;130(2):350-355.

11. Liu J, Jia L, Kan J, J in $\mathrm{CH}$. In vitro and in vivo antioxidant activity of ethanolic extract of white button mushroom (Agaricus bisporus). Food Chem Toxicol 2013;(51):310-316.

12. Jong SC, Birmingham JM. Medicinal benefits of the mushroom Ganoderma. Adv Appl Microbiol 1992;(37):101134.

13. Meschino JP. Reishi mushroom extract and immune support. Dyn Chiropractic 2002;20(12):1-8.

14. Giannenas I, Pappas IS, Mavridis S, Kontopidis G, Skoufos $\mathrm{J}$, Kyriazakis I. Performance and antioxidant status of broiler chickens supplemented with dried mushrooms (Agaricus bisporus) in their diet. Poultry Sci 2010;(89):303-311.

15. Giannenas I, Tsalie E, Chronis EF, Mavridis S, Tontis D, Kyriazakis I. Consumption of Agaricus Bisporus mushroom affects the performance, intestinal microbiota composition and morphology, and antioxidant status of turkey poults. Anim Feed Sci Techol 2011;(165):218-229.

16. Pfaller MA. Flavophospholipol use in animals: Positive implications for antimicrobial resistance based on its microbiologic properties. Diagn Microbiol Infect Dis 2006; 56(2): 115-121.

17. Niewold TA. The nonantibiotic anti-inflammatory effect of antimicrobial growth promoters, the real mode of action? A hypothesis. Poultry Sci 2007;(86):605-609.

18. Cox NA, Craven SE, Musgrove MT, Berrang ME, Stern NT. Effect of sub-therapeutic levels of antimicrobials in feed on the intestinal carriage of Campylobacter and Salmonella in turkeys. J App Poultry Res 2003; (12):32-36.

19. Dibner JJ, Richards JD. Antibiotic growth promoters in agriculture: history and mode of action. Poultry Sci 2003; (84):634-643.

20. WORLD HEALTH ORGANIZATION (WHO). First joint FAO/ OIE/WHO expert workshop on non-human antimicrobial usage and antimicrobial resistance: Scientific assessment Geneva, Switzerland, 2003. http://whqlibdoc.who.int/hq/ 2004/WHO_CDS_CPE_ZFK_2004.7.pdf. Accesed Dec 2, 2014

21. WORLD HEALTH ORGANIZATION (WHO). Second joint FAO/ OIE/WHO expert workshop on non-human antimicrobial usage and antimicrobial resistance: Management options.
Olso, Norway 2004. http://whqlibdoc.who.int/hq/2004/ WHO_CDS_CPE_ZFK_2004.8.pdf. Accesed Dec 2, 2014

22. Directive 2010/63/EU of the European Parliament and of the Council of 22 September 2010 of the protection of animals used for scientific purposes. Official J ournal of the European Union of 20th October 2010. L276/33

23. Aviagen. Ross 308 broiler: Nutrition Specification. 2007. http://www. natchix.co.za/pdf/nutrition_specifications.pdf. Accesed Dec 2, 2014.

24. Pope CR. Pathology of lymphoid organs with emphasis on immunosuppression. Vet Immunol Immunopathol 1991;30(1):31-44.

25. Guo FC, Savelkoul HFJ, Kwakkel RP, Williams BA, Verstegena MWA. Immunoactive, medicinal properties of mushroom and herb polysaccharides and their potential use in chicken diets. World Poultry Sci J 2003;59(4):427-440.

26. Borchers AT, Krishnamurthy A, Keen CL, Meyers FJ, Gershwin ME. The immunobiology of mushrooms. Exp Biol Med 2008; (233): 259-276.

27. Schroder K, Hertzog PJ, Ravasi T, Hume DA. Interferongamma: An overview of signals, mechanisms and functions. J Leuk Biol 2004; (75): 163-189.

28. Vetter J, Lelley J. Selenium level of the cultivated mushroom Agaricus bispours. Acta Aliment 2004;(33):297-301.

29. Saad MB, Gertner LR, Bona TD, Santin E. Selenium influence in the poultry immune response. Recent Pat Food Nutr Agric 2009; (1):243-247.

30. Henry PR, Ammerman CB, Campbell DR, Miles RD. Effect of antibiotics on tissue trace mineral concentration and intestinal tract weight of broiler chicks. Poultry Sci 1987;(66): 10141018.

31. Izat Al, Thomas RA, Adams MH. Effects of dietary antibiotic treatment on yield of commercial broilers. Poultry Sci 1989; (68): 651-655.

32. Izat Al, Colberg M, Reiber MA, Adams MH, Skinner JT, Cabel MC, et al. Effects of different antibiotics on performance, processing characteristics and parts yield of broiler chickens. Poultry Sci 1990;(69):1787-1791.

33. Visek WJ. The mode of growth promotion by antibiotics. J Anim Sci 1978; (46):1447-1469.

34. Rahimi S, Teymouri-Zadeh Z, Karimi-Torshizi MA, Omidbaigi $\mathrm{R}$, Rokni $\mathrm{H}$. Effect of the three herbal extracts on growth performance, immune system, blood factors and intestinal selected bacterial populations in broiler chickens. J Agric Sci Technol 2011;(13):527-539. 
